\title{
Research on Simulation of Anti-lock Braking System Based on MATLAB
}

\author{
Fengping $\mathrm{CaO}^{1, \mathrm{a}}$, Chunmei Chen ${ }^{2, \mathrm{~b}}$ and Lifa Zhou ${ }^{3, \mathrm{c}}$ \\ ${ }^{1}$ School of Shandong Jiaotong University, Jinan, 250357, China \\ ${ }^{2}$ School of Shandong Transport Vocational Collage, Weifang, 261206, China \\ ${ }^{3}$ Sinotruk (Hong Kong) Limited South Asia \& America Area, Jinan, 250101, China \\ acao2138@163.com, bailing_mm@163.com, 'zhoulf@sinotruk.com
}

Keywords: Anti-lock braking-system (ABS), Slip ratio, Vehicle model, Simulation analysis

\begin{abstract}
Anti-lock Braking System (ABS) is a system that automatically adjusts the braking force of the wheels when the vehicle is braking. It can prevent the wheels from locking to obtain the best braking effect, and improve the directional stability of the automobile. In the paper, the ABS system models based on Matlab were established, including single wheel vehicle model, tire model and vehicle braking system model. The Matlab/Simulink simulation environment was used to simulate the braking effect of a car with and without an ABS system by combining specific actual vehicle data. The simulation results confirm the validity and effectiveness of ABS.
\end{abstract}

\section{Introduction}

In recent years, cars production and ownership are increased rapidly, which brought a lot of harm to the society, such as traffic accidents [1]. In order to improve the safety of the car, an Anti-lock Braking System (ABS) is added to the conventional braking system. When the vehicle brakes, ABS automatically adjust wheel braking force of each wheel to prevent it from locking, so the braking system of automobiles can obtain the best braking effect, it is considered as one of the effective measures to enhance the driving safety of a car [2,3,4]. A simulation algorithm based on Matlab is presented in the paper to confirm the validity and effectiveness of ABS.

\section{System Model Based on Matlab}

Vehicle Dynamics Model. Taking into account the computational complexity and simulation accuracy of the vehicle model, a classic single wheel model is used, which is shown as Fig.1[5].

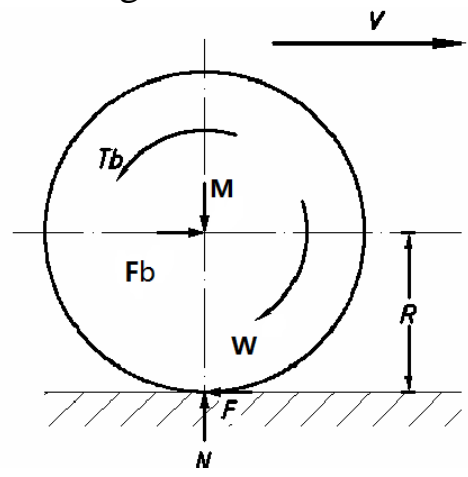

Fig.1. Single-wheel vehicle model

The required dynamic equations can be established for the two degrees of freedom of the vehicle body in the driving direction and the direction of the wheel around the main axis in the model, thus a simplified vehicle dynamic equation is obtained[5].

The motion equation of the vehicle can be described as follows:

$$
M \cdot v=-F
$$

The wheel dynamic equation can be shown as follows: 


$$
I \cdot \omega=F \cdot R-T_{b}
$$

The longitudinal friction of the wheel and braking torque can be described as follows:

$$
\begin{aligned}
& F=\mu \cdot N \\
& T_{b}=a \cdot t
\end{aligned}
$$

Where $M$ is the vehicle mass, $v$ is the vehicle speed, $F$ is the wheel friction, $I$ is the wheel moment of inertia, $R$ is the wheel radius, $\omega$ is the wheel angular velocity, $\mu$ is the road adhesion coefficient, $N$ is the ground normal reaction, $a$ is the brake effective factor, $t$ is the braking time and $T_{b}$ is the braking torque [5].

According to the above formulas, a simulation model for a single wheel vehicle subsystem can be established. The model uses the longitudinal adhesion coefficient and brake braking torque as input variables, the wheel angular velocity, vehicle body speed, and braking distance as output variables. The simulation module is shown as Fig.2.

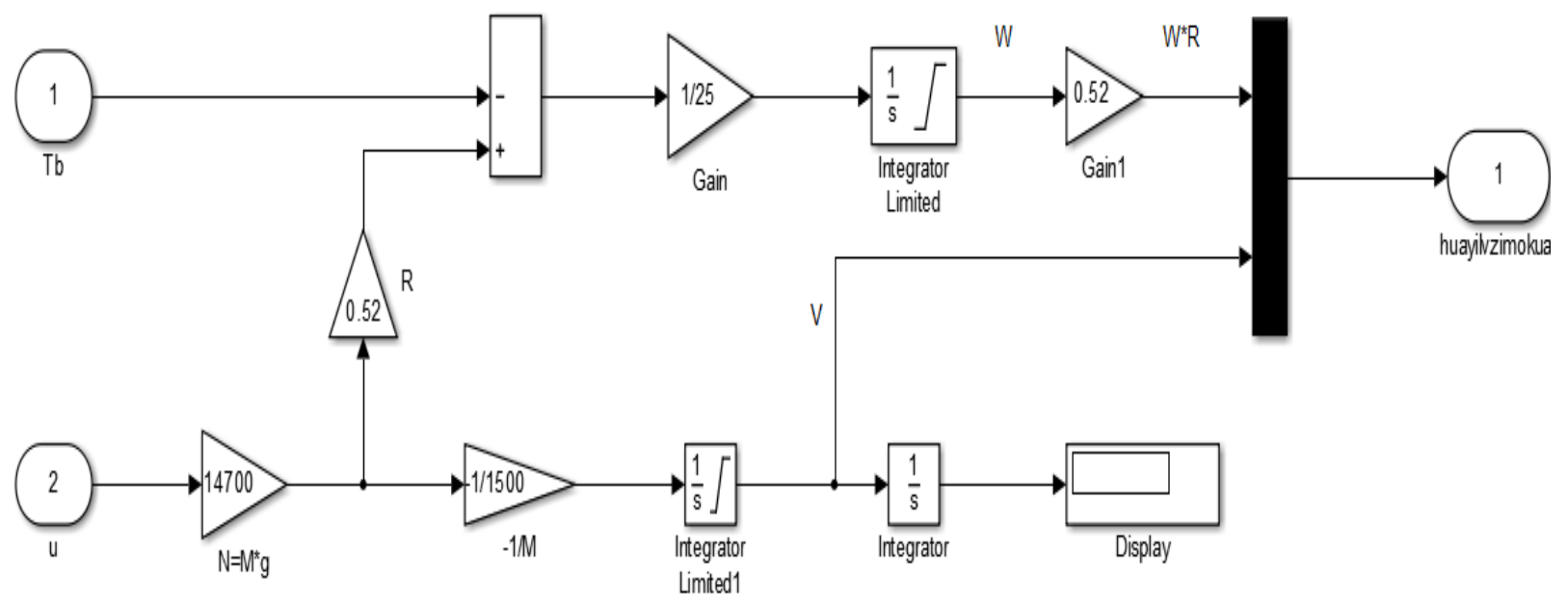

Fig.2. Vehicle dynamics model

Tire Model. Considering the research content in the paper and the accuracy of calculation, the bilinear tire model widely used in practical applications is adopted.

According to Fig.1, the mathematical expression of the bilinear tire model can be described as follows [6]:

$$
\begin{array}{cc}
\mu=\frac{\mu_{h}}{S_{o p t}} \cdot s & s<S_{o p t} \\
\mu=\frac{\mu_{h}-\mu_{g} \cdot S_{o p t}}{1-S_{o p t}}-\frac{\mu_{h}-\mu_{g}}{1-S_{o p t}} \cdot s & S \geq S_{o p t}
\end{array}
$$

Where $S_{\text {opp }}$ is the optimal slip ratio, $s$ is the slip ratio, $\mu_{h}$ is the maximum longitudinal adhesion coefficient, $\mu_{g}$ is the longitudinal adhesion coefficient when the slip ratio is $100 \%$, and $\mu$ is the longitudinal adhesion coefficient.

The simulation model of the tire model subsystem is obtained according to Equations (5) and (6). The model module takes the slip ratio as the input variable and the longitudinal adhesion coefficient as the output variable. Then the longitudinal adhesion coefficient is sent to the single wheel vehicle subsystem for simulation. And $\mu_{h}$, $S_{o p t}$, and ${ }^{\mu_{g}}$ are taken as $0.8,0.2$ and 0.7 respectively. Fig.3 is the tire simulation model.

Brake Model subsystem. The braking system consists of two parts: the transmission mechanisms and the brakes. 


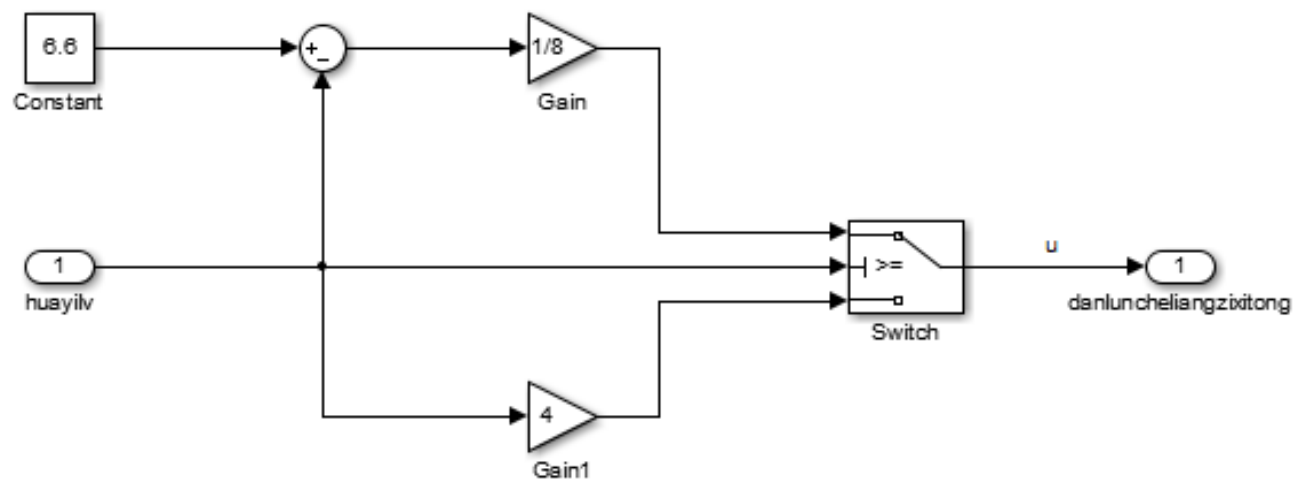

Fig.3. Tire model

The hydraulic transmission mechanism is used to simplify into a solenoid valve, taken the control signal as the input, and the flow through as the output [7]. The transfer function of the simplified model of the entire hydraulic system can be shown as follows:

$$
G(s)=\frac{100}{s \cdot(0.01 s+1)}
$$

In the brakes model, the cylinder pressure function expression of the brake torque that ultimately acts on the wheels of the vehicle can be described as follows:

$$
M_{b}=K_{p} \cdot P(t)
$$

Where $M_{b}$ is the braking torque, $K_{p}$ is the brake efficiency factor, and $P(t)$ is the brake cylinder pressure.

The simulation model of the brake model subsystem is established according to Equations 7 and 8. The model takes the control signal of hydraulic transmission mechanism as the input variable, and the braking torque as the output variable. The simulation model of the brake model subsystem is shown as Fig.4.

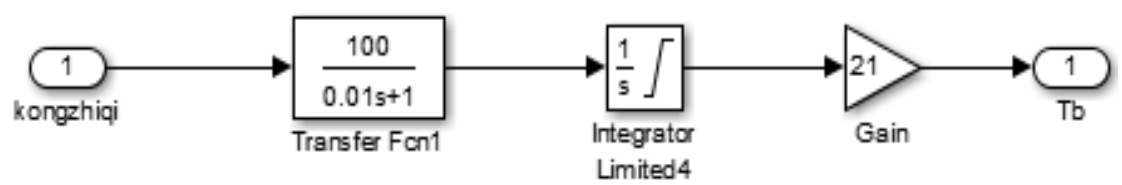

Fig.4. Brake model subsystem

Slip Rate Model. Taking the vehicle speed $\omega \cdot R$ and vehicle speed $v$ as the input variables, the slip ratio as the output variable to established the slip ratio model, which is shown as Fig.5. The slip rate is brought into the automobile tire model system for simulation.

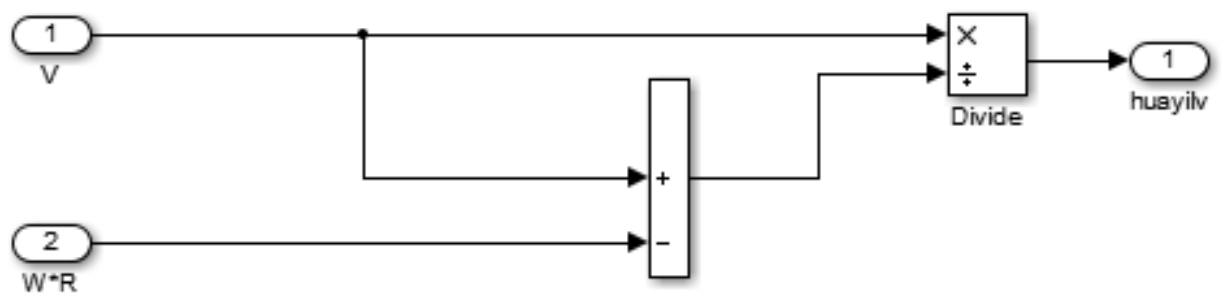

Fig.5. Slip ratio model

\section{Simulation Experiment}

Simulation Parameters. Assuming that the car is running on a dry, hard surface, the maximum adhesion coefficient is 0.9 , the sliding adhesion coefficient is 0.75 , the initial braking speed of the vehicle is $22.3 \mathrm{~m} / \mathrm{s}(80 \mathrm{~km} / \mathrm{h})$, the vehicle mass is $1500 \mathrm{~kg}$, the wheel moment of inertia is $25 \mathrm{~kg} \cdot \mathrm{m}^{2}$, the wheel radius is $0.52 \mathrm{~m}$, the simulation time is 10 seconds, and the system lag time is 0.01 second. 
According to the model established above, the braking system with or without anti-lock braking system (ABS) is simulated.

Simulation Results of Braking System Without ABS. According to the previously established quarter vehicle ABS model and the vehicle model parameters given in Table 1, the system simulation model without ABS is shown as Fig.6.

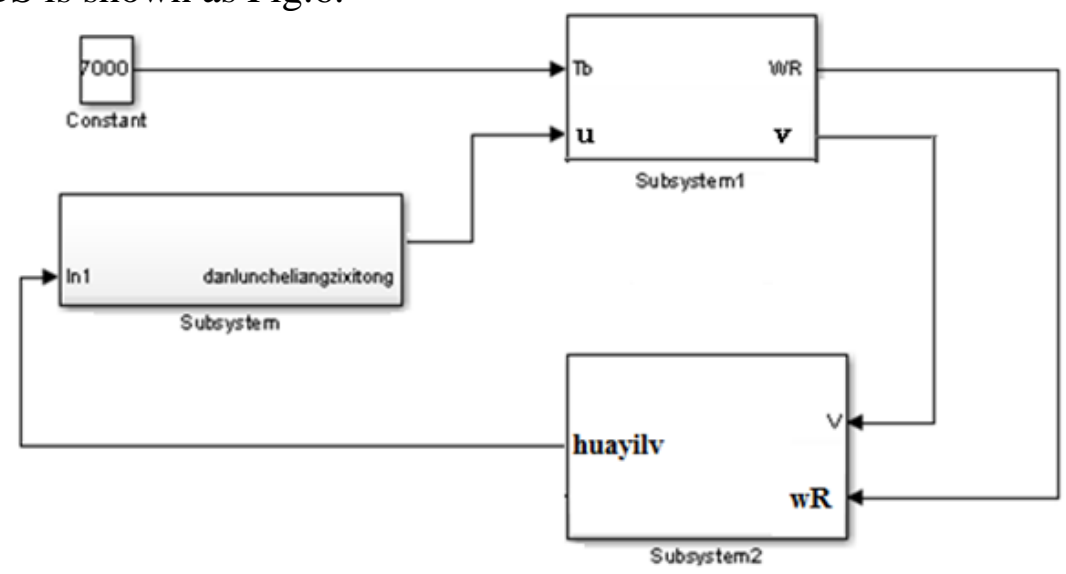

Fig.6. Simulation model of automobile braking without ABS

The simulation results of vehicle speed, wheel speed, and slip ratio during braking are shown as Fig. 7.

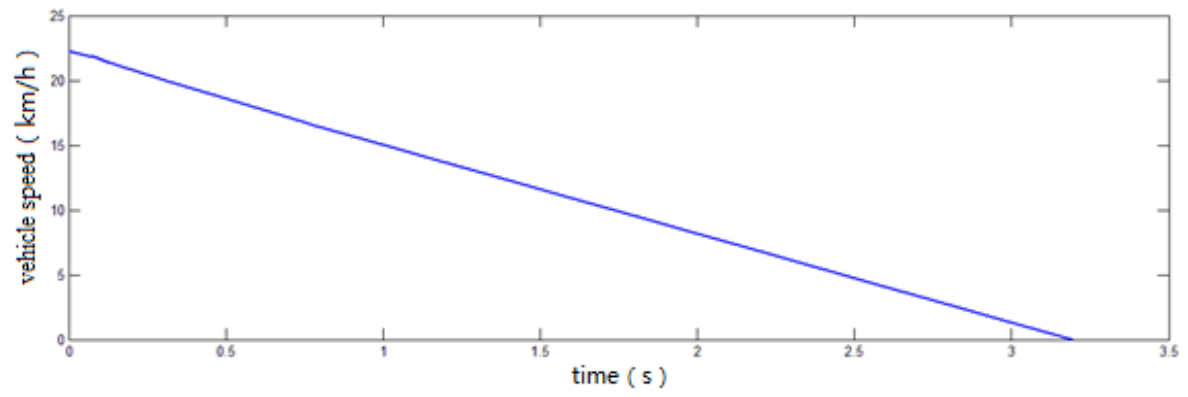

(a) vehicle speed

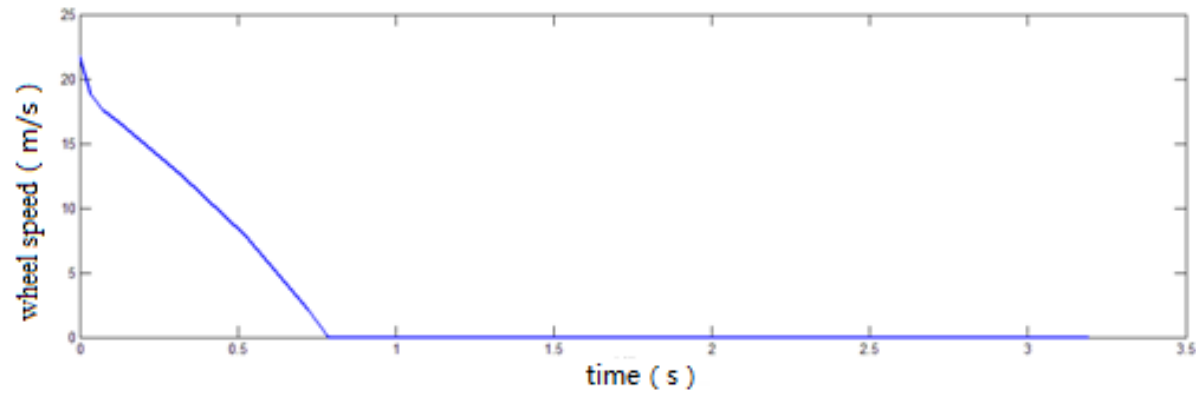

(b) wheel speed

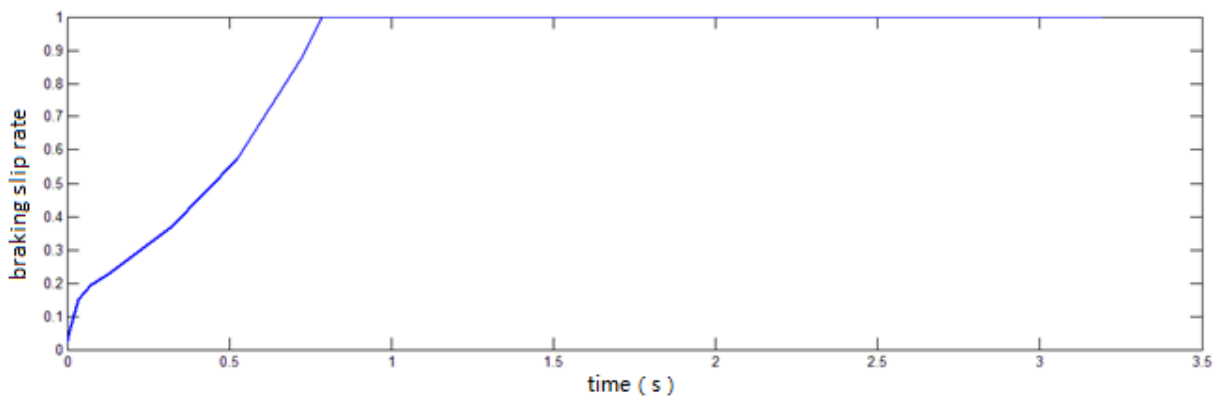

(c) braking slip rate without ABS

Fig.7. Simulation results of braking system without ABS 
Simulation Results of Braking System With ABS. According to the previously established quarter vehicle ABS model, the system simulation model with ABS is shown as Fig.8.

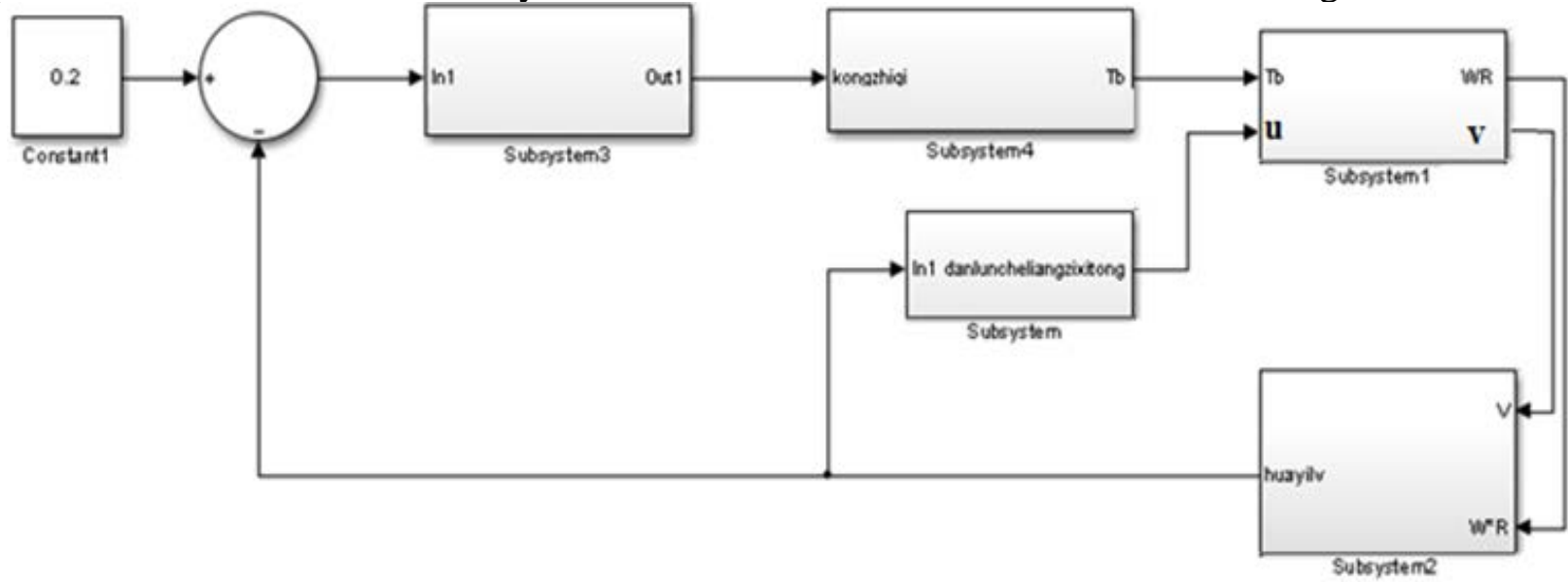

Fig. 8. Simulation model of automobile braking with ABS

Fig. 9 is the simulation results of the braking torque, wheel speed, vehicle speed, and slip ratio during braking.

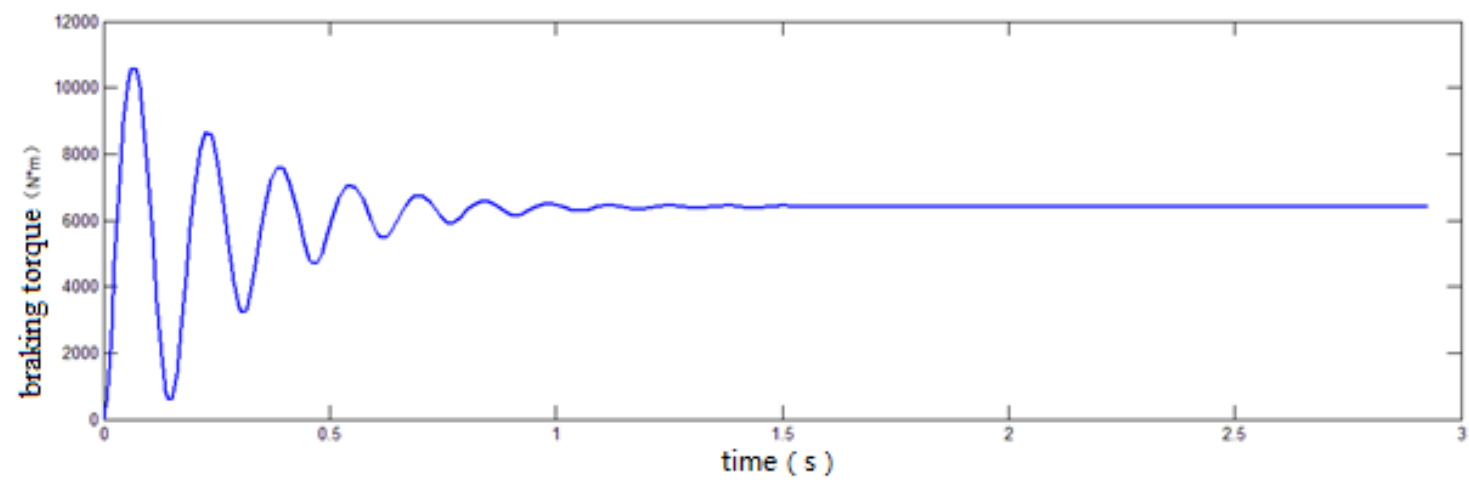

(a) braking torque

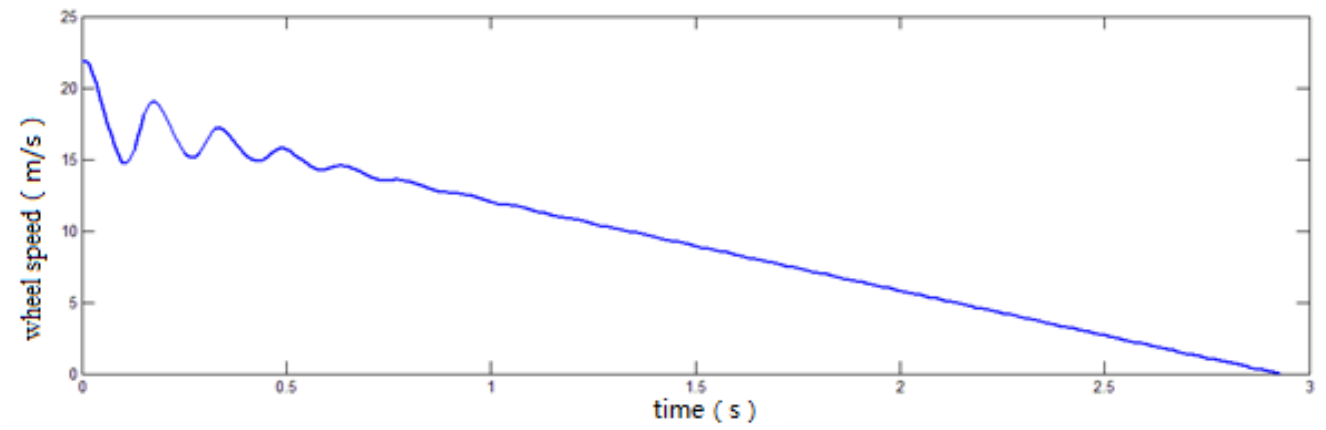

(b) wheel speed

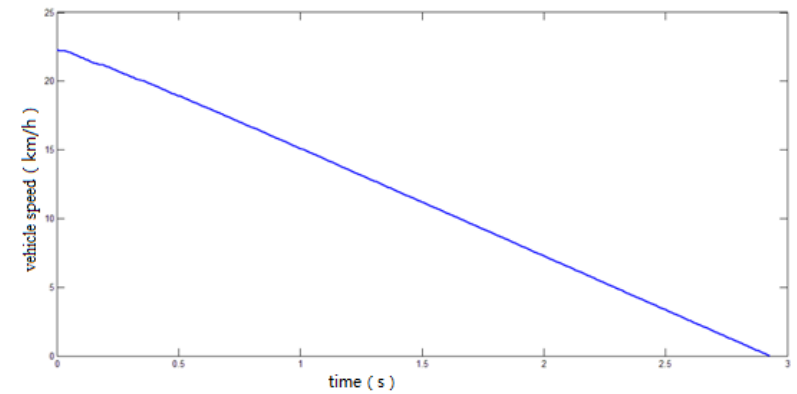

(c) vehicle speed

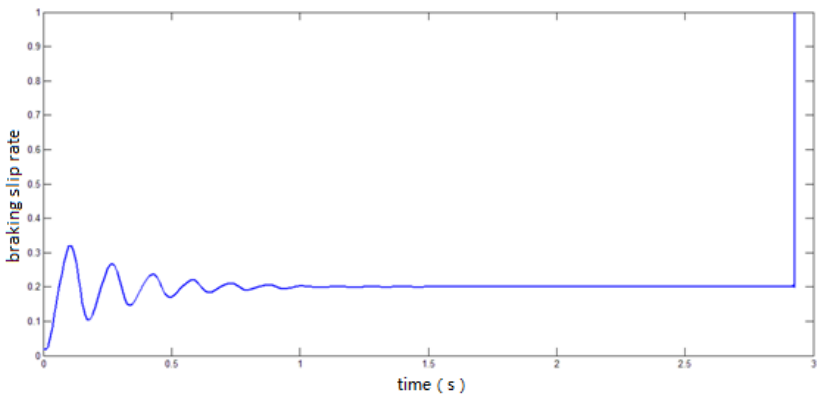

(d) braking slip rate

Fig. 9. Simulation results of braking system with ABS. 
Simulation Results Analysis. Through the simulation of the automobile brake system with ABS and without ABS, the simulation results are shown in Table 1.

Table 1 Simulation results

\begin{tabular}{|c|c|c|}
\hline Parameter & Simulation results without ABS & Simulation results with ABS \\
\hline braking time (s) & 3.23 & 2.89 \\
\hline braking distance (m) & 35.88 & 23.44 \\
\hline Wheel locking time (s) & 0.74 & 2.89 \\
\hline
\end{tabular}

Table 1 indicates that when a vehicle equipped with an ABS, the slip ratio can be well controlled in the vicinity of the optimal slip ratio, and the braking time and braking distance are shortened. The braking performance of the car has been significantly improved. Therefore, the vehicle equipped with an ABS has higher braking performance and better driving safety.

\section{Conclusions}

In the paper, a simulation algorithm of automobile braking system based on Matlab is presented. Based on the motion analysis of the vehicle braking system, a single-wheel vehicle system model was established, including a single-wheel model, a tire model, and a brake model. Then, the brake models with and without ABS were established respectively. The simulation were executed using Matlab/Simulink. The simulation results with ABS and without ABS are compared and analyzed to confirm the validity and effectiveness of the Anti-lock Braking System.

\section{Acknowledgements}

This research is supported by Natural Science Foundation of Shandong Province (ZR2015EL027), thanks to Natural Science Foundation committee of Shandong Province.

\section{References}

[1] http://www.ocn.com.cn/qiche/201801/hhhfa02174029.shtml

[2] Nobuo Iwai. Analysis on fuel economy and advanced of hybrid vehicles,JSAE Review,1999,4(2):3-11. (in Chinese)

[3] Cheng Jun. Theory and Practice of Anti-lock Braking System for Automobiles. Beijing: Beijing Institute of Technology Press, 1999. (in Chinese)

[4] Zhang Xin. Theory and Practice of Automotive Hydraulic Antilock Braking System (ABS). Changsha: Sun Yat-sen University Press,2005. (in Chinese)

[5] Yu Fan. Automotive System Dynamics. Beijing: Machinery Industry Press, 2005. (in Chinese)

[6] Yu Zhisheng. Automobile theory[M].Beijing: China Machine Press, 2009. (in Chinese)

[7] Li Yida. Control System Design and Simulation. Beijing: Tsinghua University Press, 2009.(in Chinese) 